\title{
Ballistic heat transport of quantum spin excitations as seen in $\mathrm{SrCuO}_{2}$
}

\author{
N. Hlubek, ${ }^{1}$ P. Ribeiro, ${ }^{1}$ R. Saint-Martin, ${ }^{2}$ A. Revcolevschi, ${ }^{2}$ G. Roth,${ }^{3}$ G. Behr, ${ }^{1}$ B. Büchner,${ }^{1}$ and C. Hess ${ }^{1}$ \\ ${ }^{1}$ IFW-Dresden, Institute for Solid State Research, P.O. Box 270116, D-01171 Dresden, Germany \\ ${ }^{2}$ Laboratoire de Physico-Chimie de L'Etat Solide, ICMMO, \\ UMR8182, Université Paris-Sud, 91405 Orsay, France \\ ${ }^{3}$ Institut für Kristallographie der RWTH, D-52056 Aachen, Germany
}

\begin{abstract}
Fundamental conservation laws predict ballistic, i.e., dissipationless transport behaviour in onedimensional quantum magnets. Experimental evidence, however, for such anomalous transport has been lacking ever since. Here we provide experimental evidence for ballistic heat transport in a $S=1 / 2$ Heisenberg chain. In particular, we investigate high purity samples of the chain cuprate

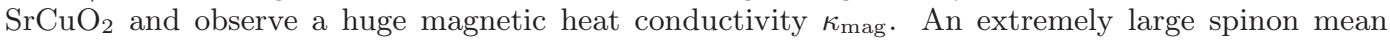
free path of more than a micrometer demonstrates that $\kappa_{\text {mag }}$ is only limited by extrinsic scattering processes which is a clear signature of ballistic transport in the underlying spin model.
\end{abstract}

PACS numbers: 75.40.Gb, 66.70.-f, 68.65.-k, 75.10.Pq

The integrability of the one-dimensional (1D) antiferromagnetic $S=1 / 2$ Heisenberg chain implies highly anomalous transport properties, in particular, a divergent magnetic heat conductivity $\kappa_{\text {mag }}$ at all finite temperatures $T \underline{\underline{1}} \underline{\underline{\underline{5}}}$ This truly ballistic heat transport suggests anomalously large life times and mean free paths of the quantum spin excitations and renders 1D quantum magnets intriguing candidates for spin transport and quantum information processing $\stackrel{\underline{6}-\underline{8}}{-}$ However, despite the rigorous prediction, experimental evidence for ballistic heat transport in quantum magnets is lacking. Nevertheless, promising large $\kappa_{\text {mag }}$ has been observed in a number of cuprate compounds which realize 1D $S=1 / 2$ Heisenberg antiferromagnets ${ }^{9}-18$ with the spin chain material $\mathrm{SrCuO}_{2}$ being a prominent example $\underline{10,18}$ although a quantitative analysis of $\kappa_{\mathrm{mag}}$ has always been difficult there since the phononic and magnetic heat conductivities are of similar magnitude at low temperature. Such experimental $\kappa_{\text {mag }}$ is always finite since extrinsic scattering processes due to defects and phonons are inherent to all materials and mask the intrinsic behavior of the chain. Formally it seems reasonable to account for the extrinsic scattering via a finite $\kappa_{\text {mag }} \sim D_{\text {th }} \tau$, where $\tau$ is a relaxation time, and $D_{\text {th }}$ represents the thermal Drude weight which (multiplied by a delta function at zero frequency) describes the intrinsic heat conductivity. In fact, it was thereby possible to identify the expected low- $T$ linearity of $D_{\mathrm{th}}(T)$ in the case of a "dirty" spin chain material where a high density of chain defects generate a large $T$-independent scattering rate $1 / \tau \underline{\underline{9}}$

In this paper we examine the heat conductivity of $\mathrm{SrCuO}_{2}$ which is considered an excellent realization of the $S=1 / 2$ Heisenberg chain $\underline{\underline{19}} \underline{21}$ Our samples of extraordinary purity allow an unambiguous separation of the phononic and magnetic contributions to the thermal conductivity. This yields the by far highest $\kappa_{\text {mag }}$ observed ${ }^{10,13}$ until now. Our analysis reveals a remarkable lower bound for the low- $T$ limit of the mean free path $l_{\text {mag }}$ of more than a micrometer. Thus our data provide striking evidence that the intrinsic heat transport of the $\mathrm{S}=1 / 2$ Heisenberg chain is indeed ballistic. With increasing temperatures $\kappa_{\text {mag }}$ is increasingly supressed due to spinon-phonon scattering which is the dominant extrinsic scattering mechanism in this material.

We have grown large single crystals of $\mathrm{SrCuO}_{2}$ by the traveling solvent floating zone method, 22 where the feed rods were prepared using the primary chemicals $\mathrm{CuO}$ and $\mathrm{SrCO}_{3}$ with both $2 \mathrm{~N}(99 \%)$ and $4 \mathrm{~N}(99.99 \%)$ purity. Cuboidal samples with typical dimensions of $(3 \times 0.5 \times 0.5) \mathrm{mm}^{3}$ were cut from the crystals, with the longest dimension parallel to the principal axes. Fourprobe measurements of the thermal conductivity $\kappa$ were performed in the $7-300 \mathrm{~K}$ range $\frac{23}{3}$ with the thermal current along the $a, b$, and $c$-axes $\left(\kappa_{a}, \kappa_{b}\right.$, and $\kappa_{c}$ respectively) for both the $2 \mathrm{~N}$ and the $4 \mathrm{~N}$ samples.

The main structural element in $\mathrm{SrCuO}_{2}$ is formed by $\mathrm{CuO}_{2}$ zig-zag ribbons, which run along the crystallographic $c$-axis (see inset Fig. 11). Each ribbon can be viewed as made of two parallel cornersharing $\mathrm{CuO}_{2}$ chains, where the straight $\mathrm{Cu}-\mathrm{O}-\mathrm{Cu}$ bonds of each double-chain structure result in a very large antiferromagnetic intrachain exchange coupling $J / k_{B} \approx 2100-2600 \mathrm{~K}$ of the $S=1 / 2$ spins at the $\mathrm{Cu}^{2+}$ sites $\frac{19,21}{2}$ The frustrated and much weaker interchain coupling $\left|J^{\prime}\right| / J \approx 0.1-0.2^{19,24}$ and presumably quantum fluctuations prevent threedimensional long range magnetic order of the system at $T>T_{N} \approx 1.5-2 \mathrm{~K} \approx 10^{-3} J / k_{B} \mathrm{~K} \stackrel{20,25}{2}$ Hence, at significantly higher $T$ the two chains within one double chain structure can be regarded as magnetically independent. In fact, low- $T$ (12 K) inelastic neutron scattering spectra of the magnetic excitations can be very well described within the $S=1 / 2$ Heisenberg antiferromagnetic chain model $\underline{21}$

Fig. 1 presents our results for $\kappa_{a}$ and $\kappa_{c}$ of $\mathrm{SrCuO}_{2}$ for both $2 \mathrm{~N}$ and $4 \mathrm{~N}$ purity as a function of $T$. We first describe the data for $2 \mathrm{~N}$ purity which are in good agreement with earlier results by Sologubenko et al $\frac{10}{21}$ A pronounced low- $T$ peak at $\sim 18 \mathrm{~K}$ with $\kappa_{\max } \approx 215 \mathrm{Wm}^{-1} \mathrm{~K}^{-1}$ is found for $\kappa_{a, 2 \mathrm{~N}}$, i.e., perpendicular to the chains. This peak and $\mathrm{a} \sim T^{-1}$-decrease at $T \gtrsim 150 \mathrm{~K}$ towards a 


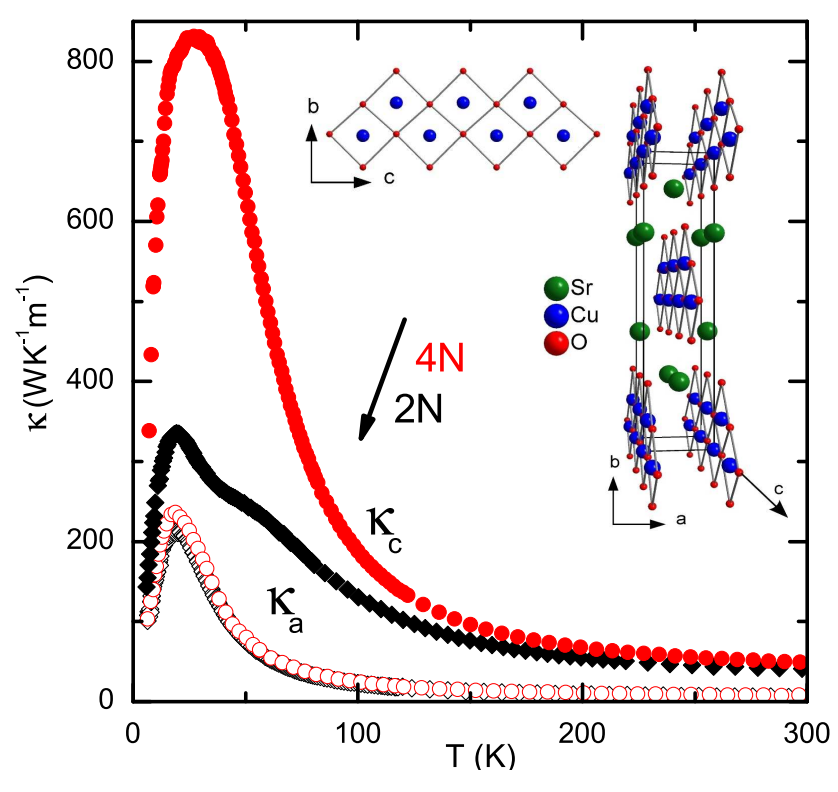

Figure 1: (Color online) $\kappa_{a}$ and $\kappa_{c}$ of $\mathrm{SrCuO}_{2}$ for different purity values. Closed (open) symbols represent $c$-axis ( $a$-axis) data, circles (diamonds) correspond to $4 \mathrm{~N}(2 \mathrm{~N})$ purity. Inset: crystal structure of $\mathrm{SrCuO}_{2}$. The symmetry is $\mathrm{Cmcm}$ with lattice constants $a=3.56 \AA, b=16.32 \AA, c=3.92 \AA .26$

small value at room temperature $\left(\sim 6 \mathrm{Wm}^{-1} \mathrm{~K}^{-1}\right)$ represent the characteristic $T$-dependence of phonon-only heat conductivity $\kappa_{\mathrm{ph}}$. The peak originates from two competing effects: $\stackrel{27}{\longrightarrow}$ at low $T$, a weakly $T$-dependent phonon mean free path $l_{\mathrm{ph}}$ and a rapidly increasing number of phonons cause $\kappa_{\mathrm{ph}}$ to increase strongly. At higher $T$, the exponentially rising number of phonon-phonon umklapp processes increasingly shortens $l_{\mathrm{ph}}$, which causes the decrease of $\kappa_{\mathrm{ph}}$. A similar low- $T$ peak (at $\sim 20 \mathrm{~K}$ ) is also present in $\kappa_{c, 2 \mathrm{~N}}$ (parallel to the chains). It is however larger $\left(\kappa_{\max } \approx 335 \mathrm{Wm}^{-1} \mathrm{~K}^{-1}\right)$ and exhibits a distinct shoulder at the high- $T$ edge $(T \gtrsim 40 \mathrm{~K})$ of the peak. $\kappa_{c, 2 \mathrm{~N}}$ decreases at higher $T$, but remains much larger than $\kappa_{a, 2 \mathrm{~N}}$ and even at room temperature $\kappa_{c, 2 \mathrm{~N}} \approx 40 \mathrm{Wm}^{-1} \mathrm{~K}^{-1}$. The apparent large anisotropy, together with the unusual $T$-dependence of $\kappa_{c, 2 \mathrm{~N}}$, is the signature of a large magnetic fraction of $\kappa_{c, 2 \mathrm{~N}}$ over a large $T$-range $\underline{10,18}$

We now turn to the new data which have been obtained for the high-purity compound. The heat transport perpendicular to the chains $\left(\kappa_{a, 4 \mathrm{~N}}\right)$ is slightly enhanced as compared to $\kappa_{a, 2 \mathrm{~N}}\left(\kappa_{\max } \approx 235 \mathrm{Wm}^{-1} \mathrm{~K}^{-1}\right)$ which reflects a somewhat reduced phonon-defect scattering. However, a much more drastic and unexpected large effect of the enhanced purity is observed in the heat transport parallel to the chains, $\kappa_{c, 4 \mathrm{~N}}$. Instead of a narrow low- $T$ peak and a shoulder as observed in $\kappa_{c, 2 \mathrm{~N}}$, a huge and broad peak centered at $\sim 28 \mathrm{~K}$ is present in $\kappa_{c, 4 \mathrm{~N}}\left(\kappa_{\max } \approx 830 \mathrm{Wm}^{-1} \mathrm{~K}^{-1}\right)$ which exceeds $\kappa_{c, 2 \mathrm{~N}}$ at $T \lesssim 70 \mathrm{~K}$ by more than a factor of 2 . Also at $70 \mathrm{~K} \lesssim T \leq 300 \mathrm{~K}$ we observe $\kappa_{c, 4 \mathrm{~N}}>\kappa_{c, 2 \mathrm{~N}}$, where interestingly both curves approach each other and at $T \gtrsim 200 \mathrm{~K}$ exhibit almost the same $T$-dependence.

Without further analysis some clear-cut conclusions can be drawn. First, the extraordinary enhancement of $\kappa_{c}$ upon the improvement of the material's purity in contrast to a concomitantly negligibly small one in $\kappa_{a}$, straightforwardly implies that the enhancement primarily concerns the magnetic heat conductivity $\kappa_{\text {mag }}$ which is present in $\kappa_{c}$ only. Second, the extreme low- $T$ sensitivity to impurities of $\kappa_{\text {mag }}$ suggests that spinon-defect scattering is the dominating process which relaxes the heat current in this regime. Third, upon rising $T$, the spinon-defect scattering is increasingly masked by a further scattering process which leads to $\kappa_{c, 2 \mathrm{~N}}$ and $\kappa_{c, 4 \mathrm{~N}}$ being very similar at $T \gtrsim 200 \mathrm{~K}$. The most reasonable candidate for this process is spinon-phonon scattering, since the only thinkable alternative, i.e. spinon-spinon scattering, is negligible $\mathrm{e}^{\frac{3-5}{5}+9}$ in this $T$-regime.

A further analysis of the data requires a reliable separation of the total measured $\kappa$ into all relevant contributions which normally add up. Since electronic contributions can be excluded in this electrically insulating material, it seems natural to assume that the measured $\kappa_{c}$ is just the sum of $\kappa_{\mathrm{mag}}$ and a phononic background $\kappa_{\mathrm{ph}, c}, \underline{\underline{9}} \underline{-14}$ where the latter can be approximated by the purely phononic heat conductivity perpendicular to the chains, $\kappa_{a} \approx \kappa_{b}$ (see inset of Fig. 22). The thus obtained $\kappa_{\text {mag }}=\kappa_{c}-\kappa_{a}$ for the $2 \mathrm{~N}$ and the $4 \mathrm{~N}$ samples are shown in Fig. 2. At $T \lesssim 35 \mathrm{~K}$, i.e., in the vicinity of the peak of $\kappa_{\mathrm{ph}, c}$, errors become large and we disregard the data in this range for further analysis. For higher $\mathrm{T}$ we account for a possible uncertainty of $\pm 30 \%$ in $\kappa_{\mathrm{ph}, c}$. Note that, in the case of the $4 \mathrm{~N}$ compound, possible errors in $\kappa_{\mathrm{mag}}$ are rendered small because obviously $\kappa_{\text {mag, } 4 \mathrm{~N}} \gg \kappa_{a, 4 \mathrm{~N}}$.

$\kappa_{\text {mag }}$ of the $4 \mathrm{~N}$ sample exhibits a sharp peak at $\sim 37 \mathrm{~K}$ with an extraordinary maximum value of about $660 \mathrm{Wm}^{-1} \mathrm{~K}^{-1}$, which is more than a factor of 3 higher than the largest reported $\kappa_{\text {mag }}, 10,13$ The peak is followed by a strong decrease upon raising $T$. Similar to the afore described typical $T$-dependence of a clean phononic heat conductor, the overall $T$-dependence of $\kappa_{\text {mag }}$ suggests that, in a simple picture, two competing effects determine $\kappa_{\text {mag }}$. The low- $T$ increase of $\kappa_{\text {mag }}$ is consistent with a regime where the effect of scattering processes is weakly $T$-dependent since $D_{\text {th }}$ is expected to increase linearly with $T \stackrel{3}{\underline{3}} \underline{-5}, \underline{9}$ The strong decrease at higher $T$ is then the result of the increasing importance of spinon-phonon scattering. $\kappa_{\text {mag }}$ of the $2 \mathrm{~N}$ sample is qualitatively very similar. However, the absolute value at the peak is much lower $(\sim 172 \mathrm{~W} / \mathrm{mK})$ and the peak's position is shifted to a higher $T(\sim 55 \mathrm{~K})$. Similarly to the $\kappa_{c}$ data, at higher $T$, the $4 \mathrm{~N}$ curve approaches that of the $2 \mathrm{~N}$ sample. The latter is consistent with the earlier notion that spinonphonon scattering is dominant at high $T$, while the differences at low $T$ suggest that spinon-phonon scattering freezes out, upon decreasing $T$, rendering spinon-defect scattering increasingly important.

We analyze $\kappa_{\text {mag }}$ quantitatively by extracting the 


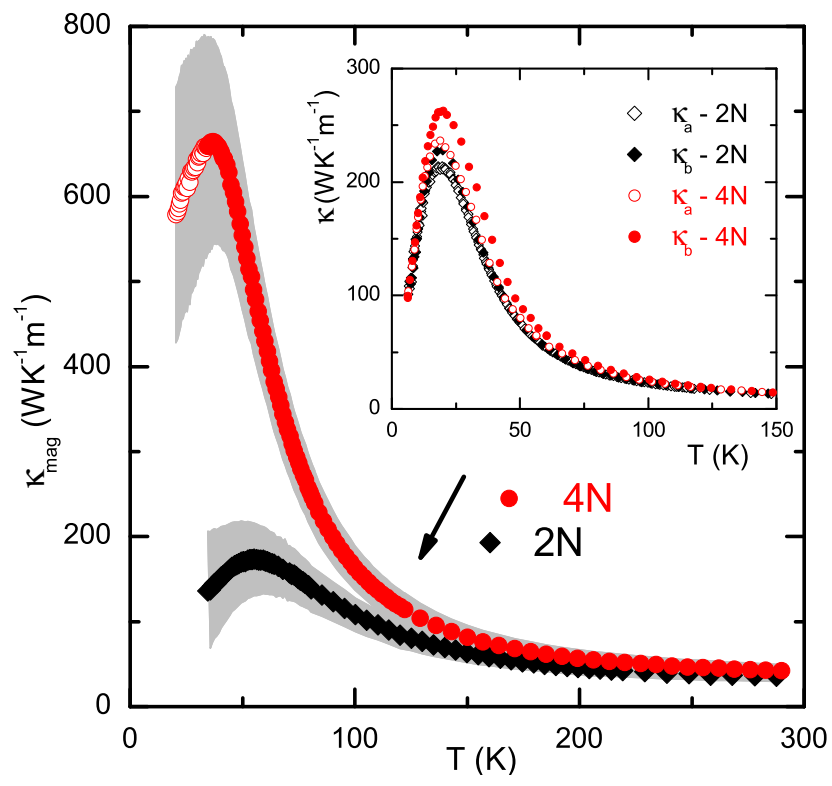

Figure 2: (Color online) $\kappa_{\text {mag }}$ of $\mathrm{SrCuO}_{2}$ for different purities. Open symbols represent low- $\mathrm{T} \kappa_{\mathrm{mag}}$ which is disregarded in the further analysis. The shaded areas show the uncertainty of the estimation of $\kappa_{\text {mag }}$ due to the phononic background. Inset: $\kappa_{a}$ and $\kappa_{b}$ perpendicular to the chain for both purities.

spinon mean free path $l_{\text {mag }}$ according to $\underline{\underline{9}} \underline{\underline{11}}$

$$
l_{\mathrm{mag}}=\frac{3 \hbar}{\pi N_{s} k_{B}^{2} T} \kappa_{\mathrm{mag}},
$$

where $N_{s}=4 / a b$ is the number of spin chains per unit area. As can be inferred from Fig. 3, $l_{\text {mag }}$ of both samples show a strong decrease with increasing $T$, which directly reflects spinon-phonon scattering becoming increasingly important. Both curves are very similar, but clear differences are present at low $T$, where $l_{\text {mag }}$ of the $2 \mathrm{~N}$ sample is somewhat lower, in accordance with a higher spinondefect scattering. We evoke Matthiesen's rule to model the $T$-dependence of $l_{\text {mag }}$ and to account for both scattering processes viz. $l_{\mathrm{mag}}^{-1}=l_{0}^{-1}+l_{\mathrm{sp}}^{-1}$. Here, $l_{0}$ describes the $T$-independent spinon-defect scattering whereas $l_{\mathrm{sp}}(T)$ accounts for the $T$-dependent spinon-phonon scattering. For the latter, we assume a general umklapp process with a characteristic energy scale $k_{B} T_{u}^{*}$ of the order of the Debye energy, which is commonly used in literature $\stackrel{10,15}{=} \mathrm{We}$ thus have

$$
l_{\mathrm{mag}}^{-1}=l_{0}^{-1}+\left(\frac{\exp \left(T_{u}^{*} / T\right)}{A_{s} T}\right)^{-1}
$$

which can be used to fit the data with $l_{0}, A_{s}$ and $T_{u}^{*}$ ( $A_{s}$ describes the coupling strength) as free parameters. We find an excellent agreement between such fits and the experimental $l_{\mathrm{mag}}$, see Fig. 3. Inspection of the fit parameters 33 yields two remarkable aspects which corroborate our previous qualitative findings. First, the parameters $A_{s}$ and $T_{u}^{*}$ which determine the spinon-phonon scattering are practically the same for both samples. In fact, an equally good fit is obtained if the same $T_{u}^{*}$ is used for both curves. Note that the extracted $T_{u}^{*} \sim 200 \mathrm{~K}$ is indeed of the order of the Debye temperature $\Theta_{D}$ of this material and thus leads to the conjecture that mostly acoustic phonons are involved in this scattering process ${ }^{34}$ Second, the spinon-defect scattering length $l_{0}$, which represents a lower bound for the low- $T$ limit of $l_{\text {mag }}$ and which should significantly depend on the sample's purity turns out to be drastically different for both cases. To be specific, we find $l_{0} \approx 300 \mathrm{~nm}$ for the $2 \mathrm{~N}$ compound and an extraordinary $l_{0} \approx 1.6 \mu \mathrm{m}$ for the $4 \mathrm{~N}$ sample, which correspond to more than 750 and 4100 lattice spacings, respectively. These findings provide a further confirmation of the above interpretation that, in both cases, $\kappa_{\text {mag }}$ is determined by the same spinon-phonon scattering process and that the difference between the two curves can be described by the different defect density only. We mention that our results are consistent with recent data by T. Kawamata et al. $\underline{35}$

A major outcome of our study is the unambiguous identification of the extrinsic scattering processes as the only relevant ones. Intrinsic spinon-spinon scattering, on the other hand, plays no role in our analysis, even in the case of the very clean sample. The strong enhancement of $\kappa_{\text {mag }}$ upon reduction of the impurity amount thus appears as the manifestation of ballistic heat transport of the underlying spin model, where $\kappa_{\text {mag }}$ is rendered finite by extrinsic scattering processes only. One might therefore speculate that $\kappa_{\text {mag }}$ of this material can be driven to much higher values in a perfect crystal.

We point out that our analysis relies on a very simple theoretical approach which was also successfully used in many other low-dimensional $S=1 / 2$ spin systems,,$\frac{9-16,28,29}{-1}$ which is surprising in view of the strong quantum nature of such systems. More sophisticated approaches might lead to a deeper understanding of the magnetic heat transport in this system on a microscopic level. In this regard it is interesting to note that, in clean samples (i.e. with large $\left.l_{0}\right), l_{\mathrm{sp}}=\left(A_{s} T\right)^{-1} \exp \left(T_{u}^{*} / T\right)$ leads to $\kappa_{c} \approx \kappa_{\operatorname{mag}} \propto \exp \left(T_{u}^{*} / T\right)$ with $T_{u}^{*} \sim 200 \mathrm{~K}$ at high $T$, in agreement with the theory proposed by Shimshoni et al $\stackrel{30}{\underline{T}}$ However, we do not observe $\kappa_{a}=\kappa_{\mathrm{ph}} \propto \exp \left(2 T_{u}^{*} / T\right)$ as expected in the same model. It seems worthwhile mentioning in this regard that the only slight enhancement of $\kappa_{a}$ observed upon increasing purity is quite unexpected. One might speculate that this is an indication of phonon scattering off the spin chains which in principle should be relevant $\underline{31,32}$

To sum up, we have investigated the spinon heat conductivity $\kappa_{\text {mag }}$ of the antiferromagnetic $S=1 / 2$ Heisenberg chain cuprate $\mathrm{SrCuO}_{2}$ for standard (99\%) and high $(99.99 \%)$ purity. The higher purity leads to a drastic enhancement of $\kappa_{\text {mag }}$ at low $T$ and we find the up-to-present by far highest reported $\kappa_{\text {mag }}$ in the high-purity sample. For higher $T$, we provide clear-cut evidence that spinonphonon scattering is the most relevant scattering which leads to a very efficient reduction of $\kappa_{\text {mag }}$. An extreme 


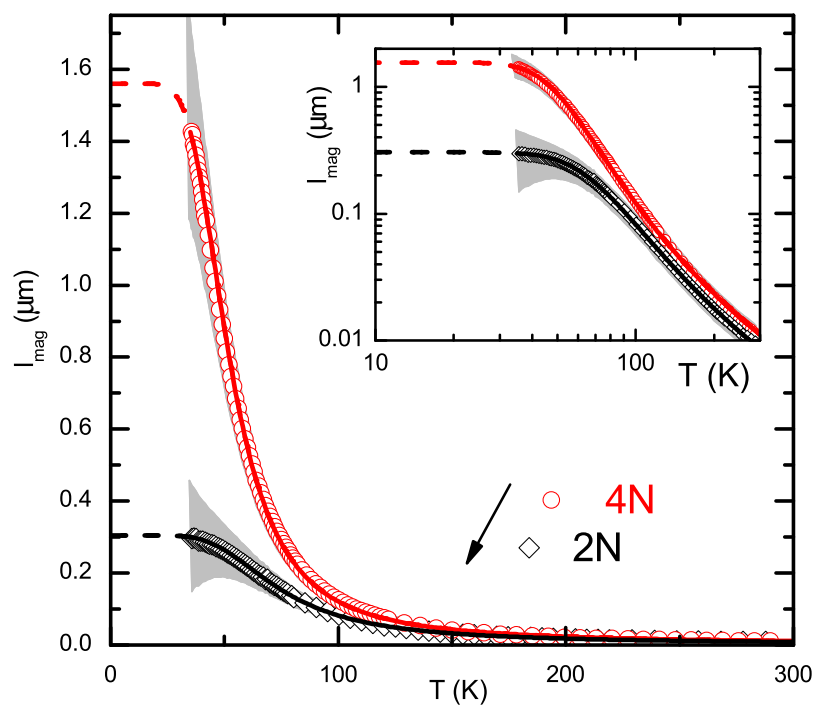

Figure 3: (color online) Magnetic mean free paths of $\mathrm{SrCuO}_{2}$ for different purities. The solid lines were calculated according to Eq. 2. The shaded area illustrates the uncertainty from the estimation of the phononic background. sensitivity of $\kappa_{\text {mag }}$ to impurities is present at low $T$, which implies that the spinon-defect scattering is dominating in this regime. A simple analysis reveals a remarkable lower bound for the low-temperature limit of the spinon mean free path $l_{\text {mag }}$ of more than a micrometer. Our results therefore suggest that $\kappa_{\text {mag }}$ is only limited by extrinsic scattering processes which appears as the manifestation of the ballistic nature of heat transport in the $S=1 / 2$ antiferromagnetic Heisenberg chain.

\section{Acknowledgments}

We thank W. Brenig, A. L. Chernyshev, S.-L. Drechsler, F. Heidrich-Meisner, P. Prelovšek, X. Zotos and A. A. Zvyagin for stimulating discussions. This work was supported by the Deutsche Forschungsgemeinschaft through grant HE3439/7, through the Forschergruppe FOR912 (grant HE3439/8) and by the European Commission through the NOVMAG project (FP6-032980).
1 X. Zotos, F. Naef, and P. Prelovsek, Phys. Rev. B 55, 11029 (1997).

2 X. Zotos, Phys. Rev. Lett. 82, 1764 (1999).

3 A. Klümper and K. Sakai, J. Phys. A: Math. Gen. 35, 2173 (2002).

4 F. Heidrich-Meisner, A. Honecker, D. C. Cabra, and W. Brenig, Phys. Rev. B 68, 134436 (2003).

${ }^{5}$ F. Heidrich-Meisner, A. Honecker, and W. Brenig, Phys. Rev. B 71, 184415 (2005).

${ }^{6}$ F. Meier and D. Loss, Phys. Rev. Lett. 90, 167204 (2003).

7 F. Meier, J. Levy, and D. Loss, Phys. Rev. Lett. 90, 047901 (2003).

8 L. F. Santos, Phys. Rev. E 78, 031125 (2008).

9 C. Hess, H. ElHaes, A. Waske, B. Buechner, C. Sekar, G. Krabbes, F. Heidrich-Meisner, and W. Brenig, PRL 98, 027201 (2007).

10 A. V. Sologubenko, K. Giannò, H. R. Ott, A. Vietkine, and A. Revcolevschi, Phys. Rev. B 64, 054412 (2001).

11 C. Hess, The European Physical Journal - Special Topics 151, 73 (2007).

12 A. V. Sologubenko, K. Giannò, H. R. Ott, U. Ammerahl, and A. Revcolevschi, Phys. Rev. Lett. 84, 2714 (2000).

13 C. Hess, C. Baumann, U. Ammerahl, B. Büchner, F. Heidrich-Meisner, W. Brenig, and A. Revcolevschi, Phys. Rev. B 64, 184305 (2001).

14 C. Hess, H. ElHaes, B. Büchner, U. Ammerahl, M. Hücker, and A. Revcolevschi, Phys. Rev. Lett. 93, 027005 (2004).

15 T. Kawamata, N. Takahashi, T. Adachi, T. Noji, K. Kudo, N. Kobayashi, and Y. Koike, Journal of the Physical Society of Japan 77, 034607 (2008).

16 C. Hess, P. Ribeiro, B. Büchner, H. ElHaes, G. Roth, U. Ammerahl, and A. Revcolevschi, Phys. Rev. B 73, 104407 (2006).

17 K. Kudo, S. Ishikawa, T. Noji, T. Adachi, Y. Koike, K. Maki, S. Tsuji, and K. Kumagai, J. Phys. Soc. Jpn.
70, 437 (2001).

18 P. Ribeiro, C. Hess, P. Reutler, G. Roth, and B. Büchner, J. Mag. Mag. Mater. 290-291, 334 (2005).

19 N. Motoyama, H. Eisaki, and S. Uchida, Phys. Rev. Lett. 76, 3212 (1996).

20 M. Matsuda, K. Katsumata, T. Osafune, N. Motoyama, H. Eisaki, S. Uchida, T. Yokoo, S. M. Shapiro, G. Shirane, and J. L. Zarestky, Phys. Rev. B 56, 14499 (1997).

21 I. A. Zaliznyak, H. Woo, T. G. Perring, C. L. Broholm, C. D. Frost, and H. Takagi, Phys. Rev. Lett. 93, 087202 (2004).

22 A. Revcolevschi, U. Ammerahl, and G. Dhalenne, Journal of Crystal Growth 198/199, 593 (1999).

23 C. Hess, B. Büchner, U. Ammerahl, and A. Revcolevschi, Phys. Rev. B 68, 184517 (2003).

24 T. M. Rice, S. Gopalan, and M. Sigrist, Europhys. Lett. 23, 445 (1993).

25 I. A. Zaliznyak, C. Broholm, M. Kibune, M. Nohara, and H. Takagi, Phys. Rev. Lett. 83, 5370 (1999).

26 L. Teske and H. Müller-Buschbaum, Z. Anorg. Allg. Chem. 379, 234 (1971).

27 R. Berman, Thermal Conduction in Solids (At the Clarendon Press, Oxford, 1976).

28 C. Hess, B. Büchner, U. Ammerahl, L. Colonescu, F. Heidrich-Meisner, W. Brenig, and A. Revcolevschi, Phys. Rev. Lett. 90, 197002 (2003).

29 C. Hess, C. Baumann, and B. Büchner, J. Mag. Mag. Mater. 290-291, 322 (2005).

30 E. Shimshoni, N. Andrei, and A. Rosch, Phys. Rev. B 68, 104401 (2003).

31 A. L. Chernyshev and A. V. Rozhkov, Phys. Rev. B 72, 104423 (2005).

32 A. V. Rozhkov and A. L. Chernyshev, Phys. Rev. Lett. 94, 087201 (2005).

${ }^{33}$ For the $4 \mathrm{~N}$ and $2 \mathrm{~N}$ compounds we get $l_{0,4 N}=$ 
$(1.56 \pm 0.16) \mu \mathrm{m}, \quad T_{u, 4 N}^{*}=(204 \pm 11) \mathrm{K}, \quad A_{s, 4 N}=$ $(58.6 \pm 5.4) \quad 10^{-16} \mathrm{~m} / \mathrm{K}$ and $l_{0,2 N}=(305 \pm 5) \mathrm{nm}$, $T_{u, 2 N}^{*}=(217 \pm 3) \mathrm{K}, A_{S, 2 N}=(78 \pm 1) 10^{-16} \mathrm{~m} / \mathrm{K}$, respectively. Setting $T_{u, 2 N}^{*}=T_{u, 4 N}^{*}=204 \mathrm{~K}$ after fitting the $4 \mathrm{~N}$ data gives $l_{0,2 N}=(320 \pm 13) \mathrm{nm}$ and $A_{s, 2 N}=(72 \pm 5) 10^{-16} \mathrm{~m} / \mathrm{K}$ for $2 \mathrm{~N}$. The errors account for the accuracy of the fit. It is also possible to obtain a good fit with the same $A_{s}$ for the $4 \mathrm{~N}$ and $2 \mathrm{~N}$ cases. However, individual $A_{s}$ account for errors in the absolute value, while fixing the energy scale by $T_{u}^{*}$ seems physically justified.

34 Modeling $l_{\text {mag }}$ with $l_{\mathrm{sp}} \propto \exp \left(T^{*} / T\right)$ which accounts for the alternative scenario of spinons scattering off optical phonons ${ }^{29}$ results in a fit of similar quality with comparable $l_{0}$ and $T^{*} \sim 300 \mathrm{~K}$.

35 T. Kawamata, N. Kaneko, M. Uesaka, M. Sato, and Y. Koike, unpublished data. 